\title{
Um Procedimento Teórico- Experimental para a Estimativa da Difusividade e Condutividade Térmica de Alimentos em Pastas
}

Laís S. de Moura, Ana C. Teixeira, Nathália G. Cardoso, Orlene S. Costa, Valter H. C. Silva, José S. Sales \& Heibbe C. B. de Oliveira

O conhecimento das propriedades térmicas é de grande importância para simulação da variação da temperatura em sistemas de transporte, estocagem e dimensionamento industrial, principalmente na indústria de alimentos. Neste trabalho nós propomos uma metodologia teórica-experimental para a predição da difusividade e condutividade térmica de alimentos baseada em um procedimento híbrido entre um aparato experimental muito simples e o método de otimização Levenberg-Marquardt. Para validar esta metodologia nós utilizamos pasta de tomate comercial. A difusividade e a condutividade térmica obtidas foram $0,093 \mathrm{~cm}^{2} / \mathrm{min}$ e $0,544 \mathrm{~W} / \mathrm{m}$.K, respectivamente. Estes resultados estão de acordo com a literatura.

Palavras-chave: pasta de tomate, difusividade térmica, condutividade térmica.

The knowledge of thermal properties is very important for simulation of temperature variation in transport, storage system and industrial sizing, mainly in food industry. In this work we propose a theoretical-experimental methodology for prediction of thermal diffusivity and conductivity of foods based on hybrid procedure between a very simple experimental apparatus and the Levenberg-Marquardt optimization method. To validate this procedure we used commercial tomato paste. The diffusivity and conductivity thermal obtained were $1.55 .10^{-7} \mathrm{~m}^{2} / \mathrm{s}$ e $0.544 \mathrm{~W} / \mathrm{m} . \mathrm{K}$, respectively. These results are in close agreement with literature data.

Keywords: tomato paste, thermal diffusivity, thermal conductivity. 


\section{Introdução}

As propriedades térmicas são essenciais para a eficiência e controle das operações de processamento de alimentos que envolvem transferências de calor. Essas propriedades são necessárias para simular a temperatura durante tratamentos, transporte, estocagem e distribuição. Entre elas pode-se citar a difusividade térmica, condutividade térmica, calor específico e massa específica. Essas propriedades variam de acordo com a composição química, umidade e porosidade, por exemplo, do material analisado (Betta et al, 2009; Sousa, 2009) ${ }^{1-16}$. O conhecimento da difusividade térmica é importante quando se deseja determinar a evolução temporal em sistemas submetidos às transformações térmicas. Essa propriedade está intimamente ligada à dinâmica de penetração de calor em alimentos. Ela pode ser determinada por várias metodologias, como por exemplo: i) soluções numéricas; ii) aproximação analítica; e iii) método de Sweat, que faz a aplicação direta da equação:

$$
\alpha=\frac{k}{\rho c_{p}},
$$

onde a difusividade térmica $(\alpha)$ está em função da condutividade térmica $(k)$, calor específico a pressão constante $\left(c_{p}\right)$ e massa específica $(\rho)$ (Carbonera et al., 20035; Baïri et al., 2007,2, Huang e Liu, 2009 ${ }^{11}$; Carciofi et al., 2002 ; Garrote et al, 2000 ${ }^{10}$; Erdoğdu, 2005 ; Silans et al., 2006 ${ }^{15}$ ).

O conhecimento da condutividade térmica também é relevante e encontra aplicação em quase todas as áreas de processamento de alimentos, como por exemplo, a secagem, a aeração e o resfriamento de grãos (Park et al., $1997)^{14}$. Se há um gradiente de temperatura entre duas superfícies através do qual o calor flui, a quantidade de calor (q) que irá fluir em uma unidade de tempo ( $\mathrm{t}$ ) por certa espessura (L), através de uma unidade de área (A), é chamada de condutividade térmica, denominada por $\mathrm{k}$, definida como segue:

$$
q=-K\left(\frac{A}{L}\right)\left(\frac{d t}{d x}\right)
$$

Por outro lado, ela pode ser determinada mais facilmente utilizando-se a Equação 1.
A determinação da difusividade térmica é possível a partir da solução da seguinte equação íntegro-diferencial:

$$
\nabla^{2} T+\frac{q}{k}=\frac{1}{\alpha} \frac{\partial T}{\partial t^{\prime}}
$$

a qual é originária de um balanço de energia proveniente da Lei de Fourier para transferência de calor por condução. Na Equação 3, o primeiro termo expressa o gradiente de temperatura, o segundo a fonte térmica interna do sistema e o terceiro representa a variação temporal da temperatura. Neste trabalho, propomos um procedimento híbrido teórico-experimental para a estimativa da difusividade e condutividade térmica em alimentos baseada na equação de transferência de calor longitudinal a qual é resolvida pelo método de regressão não-linear de Levenberg - Marquardt $(\mathrm{LM})^{12,13}$.

\section{Material e métodos}

Este trabalho consistiu em quatro etapas distintas: 1) construção e montagem do sistema de transmissão de calor; 2) ensaio de transferência de calor; 3) definição do modelo matemático representativo da transferência de calor por condução; 4) ajuste computacional do modelo matemático aos dados experimentais; e 5) estimativa dos erros entre os dados experimentais e os dados previstos pelo modelo matemático.

\section{CONSTRUÇÃO E MONTAGEM DO SISTEMA DE TRANSMISSÃO DE CALOR}

Um tubo de PVC (policloreto de vinila) de $3,5 \mathrm{~cm}$ diâmetro e 19,0 cm de comprimento foi perfurado com quatro furos, equidistantes $4,0 \mathrm{~cm}$, constituindo as seguintes posições longitudinais da base até o topo: $Z_{1}=$ $4,0 \mathrm{~cm}, \mathrm{Z}_{2}=8,0 \mathrm{~cm}, \mathrm{Z}_{3}=12,0 \mathrm{~cm}$; e $\mathrm{Z}_{4}=16,0 \mathrm{~cm}$, como demonstrado na Figura 1.

A base do tubo de PVC foi vedada com papel alumínio e sua lateral foi revestida com uma camada de cortiça de quatro milímetros de espessura a fim de isolálo termicamente. A base de papel alumínio teve o objetivo de conter o material a ser introduzido no tubo e permitir a transferência de calor com baixa resistência térmica. Tanto a base de papel alumínio quanto o revestimento de cortiça tiveram a finalidade de promover a transmissão de calor na direção longitudinal. 


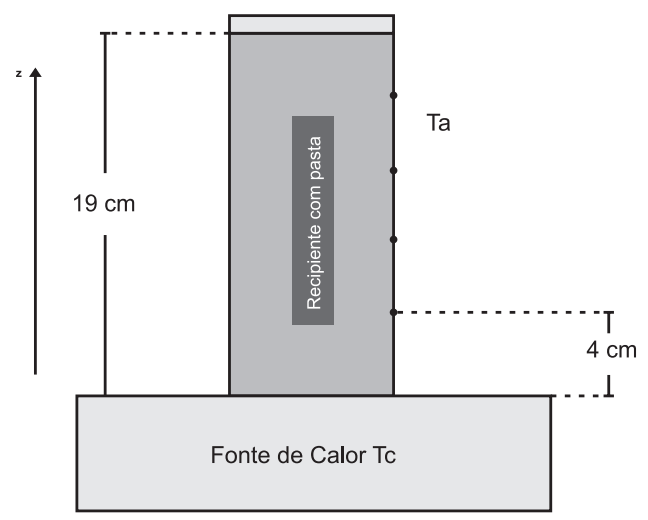

Figura 1. Desenho esquemático do sistema de transferência de calor.

A pasta de tomate comercial foi pesada em um volume pré-estabelecido a fim de se determinar sua massa específica. Em seguida, sem nenhum tratamento prévio, a pasta de tomate foi introduzida no tubo até total preenchimento. Uma tampa de isopor foi colocada no topo, como o objetivo de evitar a condensação de vapores. Logo após, todo o tubo foi recoberto com papel alumínio para minimizar os efeitos da radiação térmica. Finalmente, quatro termômetros digitais foram instalados nos furos dispostos ao longo do tubo.

\section{ENSAIO DE TRANSFERÊNCIA DE CALOR}

Inicialmente, registraram-se as temperaturas medidas pelos quatro termômetros acoplados ao tubo contendo pasta de tomate comercial e a temperatura do ar. O ensaio de transferência de calor por condução unidimensional e em regime transiente foi conduzido colocando-se a superfície da base do tubo, com papel alumínio, em contato com um banho termostatizado, mantido a uma temperatura de $50^{\circ} \mathrm{C}$ (Figura 2). A partir deste instante mediu-se a variação de temperatura durante 125 minutos em intervalos de 5 minutos.

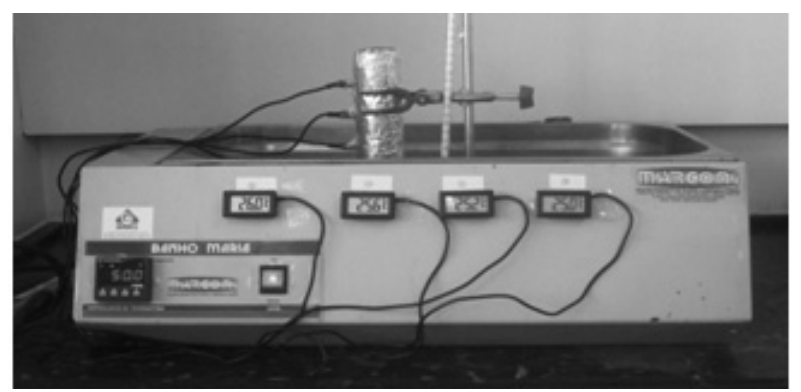

Figura 2. Esquema do experimento

\section{DEFINIÇÃO DO MODELO MATEMÁTICO REPRESENTATIVO DA TRANSFERÊNCIA DE CALOR POR CONDUÇÃO}

Como o sistema de transferência de calor por condução apresenta um volume de controle cilíndrico, uma área de transmissão de calor de seção circular e o fluxo de transporte de calor ocorrem em regime transiente. Assim a Equação (3) pode ser re-escrita tridimensionalmente em coordenadas cilíndricas, conforme:

$$
\frac{1}{R} \frac{\partial}{\partial R}\left(R \frac{\partial T}{\partial R}\right)+\frac{1}{R^{2}} \frac{\partial^{2} T}{\partial \varphi^{2}}+\frac{\partial^{2} T}{\partial z^{2}}+\frac{q}{k}=\frac{1}{\alpha} \frac{\partial T}{\partial t} .
$$

Por sua vez, como o volume cilíndrico é isolado termicamente nas laterais, forçando a condução de calor unidirecional no eixo $\mathrm{z}$, e assumindo que não existe uma fonte térmica interna ao sistema, a Equação (4) é simplificada para:

$$
\frac{\partial^{2} T}{\partial z^{2}}=\frac{1}{\alpha} \frac{\partial T}{\partial t^{\prime}}
$$

sendo $\mathrm{T}$ a temperatura, $\mathrm{z}$ a direção longitudinal, $\mathrm{t}$ o tempo e $\alpha$ a difusividade térmica.

A solução para a Equação (5) foi proposta por Fourier em seu tratado Théorie Analytique de la Chaleur, publicado em $1822^{12}$. Boyce e DiPrima (2000) $)^{12,13}$ também apresentaram uma solução completa, expressa por:

$$
T(z, t)=T_{s}+\sum_{n=1}^{\infty} c_{n} e^{-n^{2} \pi^{2} \alpha t / L^{2}} \operatorname{sen}\left(\frac{n \pi z}{L}\right),
$$

sendo $T_{s}$ a temperatura da fonte térmica e $c_{n}$ é o coeficiente de Fourier, que é baseado nas seguintes condições de contorno:

$$
T(z, 0)=T(0, t)=T_{a} .
$$

Aplicando a transformada de Fourier, descobriu-se então o coeficiente $c_{n}$ é dado por:

$$
c_{n}=\frac{2}{L} \int_{0}^{L}\left(T_{a}-T_{s}\right) \operatorname{sen}\left(\frac{n \pi z}{L}\right) d x=\frac{4}{(2 n+1) \pi}\left(T_{a}-T_{s}\right) .
$$

Finalmente, obteve-se a equação completa da distribuição de temperatura, conforme:

$$
T(z, t)=T_{s}+\sum_{n=0}^{\infty}\left\{\frac{4}{(2 n+1) \pi}\left(T_{a}-T_{s}\right) e^{-(2 n+1)^{2} \pi^{2} \alpha t / L^{2}} \operatorname{sen}\left[\frac{(2 n+1) \pi z}{L}\right]\right\}
$$




\section{AJUSTE COMPUTACIONAL DO MODELO MATEMÁTICO AOS DADOS EXPERIMENTAIS}

O modelo matemático não linear, representativo da distribuição de temperatura para transferência de calor por condução unidimensional e em regime transiente, expresso pela Equação (9), foi ajustado aos dados experimentais obtidos, onde se determinou o valor de $\alpha$ (difusividade térmica).

É importante pontuar que a difusividade térmica é o único parâmetro a ser obtido por essa técnica. Essa determinação é possível pelo uso do algoritmo de Levenberg-Marquardt. Conforme pode ser visto na Equação (9), a sua descrição matemática envolve uma quantidade infinita de termos. No entanto, para tratarmos o sistema real em questão é necessário truncar a série. Neste caso optamos por usar cinco, nove e doze termos. A partir do ajuste computacional do modelo matemático via método de Levenberg-Marquardt a difusividade térmica foi obtida e, conseqüentemente, a estimativa da condutividade térmica da pasta de tomate foi feita pelo método de Sweat.

Abaixo mostramos um pseudocódigo do procedimento computacional adotado neste trabalho durante o procedimento de minimização da função,

$$
\chi^{2}=\frac{1}{N} \sum_{i=1}^{N}\left(T(z, \mathrm{t})_{e x p}-T(z, \mathrm{t})_{T e o r}\right)^{2}:
$$

I inicializa-se o procedimento com um valor para a difusividade térmica;

ii em seguida usa-se o método Levenberg-Marquardt para gerar um novo valor para a difusividade térmica;

iii calcula-se a Equação (9);

iv calcula-se o valor da função $\chi^{2}$ usando todos os pontos experimentais $\mathrm{T}(\mathrm{z}, \mathrm{t})$,

$\mathbf{v}$ compara-se os valores de $\chi^{2}$ para os dois últimos conjuntos de parâmetros:

- se o $\chi^{2}$ para o último conjunto de parâmetros for menor do que a anterior, então se aceita este valor de $\alpha$;

- caso contrário, mantém o valor de $\alpha$ como ponto de partida para gerar um novo $\alpha$;

vi volta-se ao passo (ii) para gerar a nova função $\chi^{2}$;

vii assim, este procedimento entre os passos (ii) e (v) é repetido até atingir o valor mínimo do desvio quadrático médio, obedecendo a um dado critério de convergência, inicialmente pré-estabelecido.

\section{ESTIMATIVA DO ERRO ENTRE OS DADOS EXPERIMENTAIS E OS DADOS PREVISTOS}

Os erros relativos entre os dados experimentais da variação espacial e temporal da temperatura e os dados obtidos pelo modelo matemático, expressos pela Equação (9), para doze termos na construção deste modelo foram estimados e dispostos em gráficos em função do tempo, visando verificar o comportamento da dispersão dos erros. Os erros foram calculados usando a seguinte relação:

$$
\text { Erro Relativo }=\left(\frac{T_{\text {exp }}-T_{\text {teor }}}{T_{\exp }}\right) .
$$

\section{Resultados e discussão}

Os perfis de temperatura dos quatro termômetros digitais, ilustrado na Figura 3, retratam a dispersão da distribuição da temperatura em função do tempo para transferência de calor por condução em pasta de tomate comercial. Observa-se que quanto mais próximo o termômetro estiver da fonte aquecedora, maior é a curvatura do perfil de temperatura e mais brevemente ela tende a atingir a temperatura de equilíbrio.

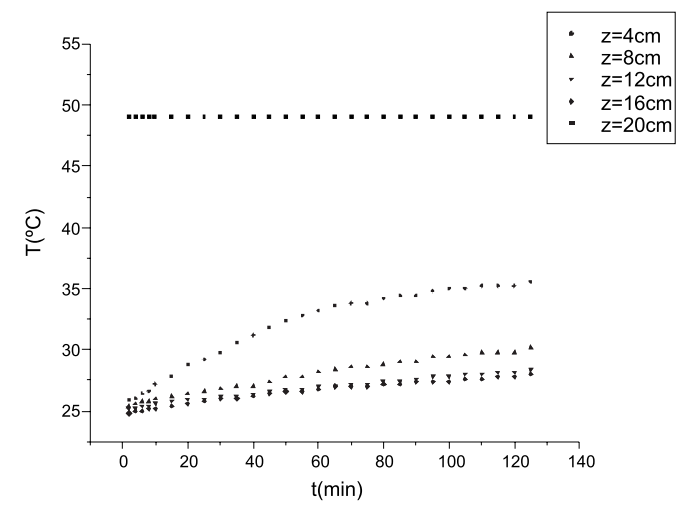

Figura 3. Variação espacial e temporal da temperatura em pasta de tomate comercial

Os valores da difusividade térmica preditos pelo ajuste do modelo matemático não linear estão apresentados na Tabela 1, para as simulações computacionais com cinco, nove e doze termos na construção da Equação (9).

A avaliação dos valores $\alpha$ obtidos pelo método computacional de Levenberg-Marquardt mostra que o aumento dos termos da Equação (9) não leva a 
uma alteração significativa na difusividade térmica $(\alpha)$, isto é, cinco termos na construção da Equação (9) são suficientes para descrever a transferência de calor no sistema. O emprego desta nova metodologia determinou uma difusividade térmica para pasta de tomate comercial em torno de $1,55.10^{-7} \mathrm{~m}^{2} \cdot \mathrm{s}^{-1}$, com um valor médio para o $\chi^{2}$ de $1,86\left({ }^{\circ} \mathrm{C}\right)^{2}$. Este valor está de acordo com as seguintes literaturas ${ }^{1-5}: 1$ ) cujo valor de $\alpha$ foi obtido numa faixa de $1,30-1,63 \cdot 10^{-7} \mathrm{~m}^{2} \cdot \mathrm{s}^{-1} ; 2$ ) que obtiveram o valor de $1,42.10^{-7} \mathrm{~m}^{2} \cdot \mathrm{s}^{-1}$; e 3), sendo $\alpha=2,10 \cdot 10^{-7} \mathrm{~m}^{2} \cdot \mathrm{s}^{-1}$. Estes resultados indicam que a metodologia proposta neste trabalho descreve bem o fenômeno de condução de calor em matérias pastosas ou cremosas, obtendo assim valores para a difusividade térmica em bom acordo com dados da literatura.

Tabela 1. Difusividade térmica $(\alpha)$ e o erro $\chi 2$ para o experimento obtido através do método de Levenberg - Marquardt.

\begin{tabular}{|c|c|c|}
\hline $\begin{array}{c}\text { No. de Termos } \\
\text { da Equação (9) }\end{array}$ & $\begin{array}{c}\boldsymbol{\alpha}, \mathbf{m}^{2} \cdot \mathbf{s}^{\mathbf{1}} \text { (obtido via método } \\
\text { de Levenberg-Marquardt) }\end{array}$ & $\boldsymbol{\chi}^{2},{ }^{\mathbf{0}} \mathbf{C}^{\mathbf{2}}$ \\
\hline 5 & $1,550.10^{-7}$ & 1,84 \\
\hline 9 & $1,555.10^{-7}$ & 1,88 \\
\hline 12 & $1,553.10^{-7}$ & 1,87 \\
\hline
\end{tabular}

A Figura 4 ilustra o diagrama de dispersão dos erros relativos entre dados obtidos experimentalmente e os dados teóricos, previstos pelo modelo da Equação (9) com doze termos.

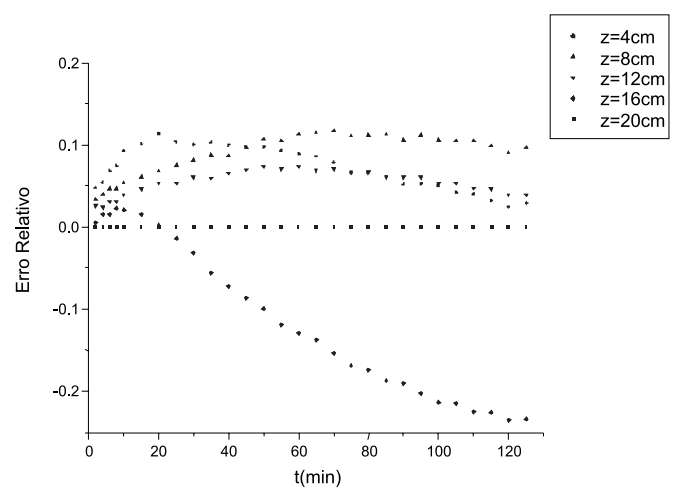

Figura 4. Diagrama de erros entre os dados experimentais e os dados obtidos por meio do modelo matemático.

A determinação da massa específica ( $\rho$ ) da pasta de tomate comercial, igual a 1,062 kg.m ${ }^{-3}$, possibilitou a estimativa da condutividade térmica $(\mathrm{k})$ por meio da Equação (1). O calor específico ( $\mathrm{c}_{\mathrm{p}}$ ) adotado para estimativa de $\mathrm{k}$, foi o valor determinado por Drusas e Saravacos (1985), igual a 3,302 $\mathrm{J}_{\mathrm{kg}}{ }^{-1} \cdot \mathrm{K}^{-1}$. Deste modo, o valor estimado da condutividade térmica foi igual a $0,544 \mathrm{~W} \cdot \mathrm{m}^{-1} \cdot \mathrm{K}^{-1}$, valor em bom acordo com o encontrado na literatura equivalente ${ }^{7}$ a $0,525 \mathrm{~W} \cdot \mathrm{m}^{-1} \cdot \mathrm{K}^{-1}$.

\section{Conclusões}

A metodologia proposta neste trabalho mostrou-se bastante viável, pois, além de simples, possui um baixo custo e é de fácil execução, além de ser também eficaz na determinação da difusividade térmica de pastas, já que os resultados obtidos foram próximos aos valores encontrados na literatura. $\mathrm{O}$ valor da difusividade térmica $(\alpha)$ estimado para pasta de tomate comercial foi igual a $1,55.10^{-7} \mathrm{~m}^{2} \cdot \mathrm{s}^{-1}$, muito próximo aos valores $\mathrm{s}^{5-7}: 1$ ) Da faixa $1,30-1,63 \cdot 10^{-7} \mathrm{~m}^{2} \cdot \mathrm{s}^{-1}$; 2) $\alpha=1,42 \cdot 10^{-7} \mathrm{~m}^{2} \cdot \mathrm{s}^{-1}$; e 3) $\alpha=2,10 \cdot 10^{-7} \mathrm{~m}^{2} \cdot \mathrm{s}^{-1}$.

Por sua vez, a estimativa do valor da condutividade térmica (k) da pasta de tomate comercial também foi bem sucedida, já que esta depende do valor da difusividade térmica, apresentando um valor de $0,544 \mathrm{~W} \cdot \mathrm{m}^{-1} \cdot \mathrm{K}^{-1}$, valor próximo ao disponibilizado por (DRUSAS e SARAVACOS, 1985) ${ }^{7}$, que obteve $\mathrm{k}=0,525 \mathrm{~W} \cdot \mathrm{m}^{-}$ ${ }^{1} \cdot \mathrm{K}^{-1}$ para massa de tomate. O sucesso desta aplicação metodológica é fortemente alicerçado pelo fato de que o erro relativo e o erro $\chi^{2}$ estarem em torno de 0,02097 e $1,86\left({ }^{\circ} \mathrm{C}\right)^{2}$, respectivamente.

\section{Agradecimentos}

À Pró-Reitoria de Pesquisa e Pós-Graduação (PrP) da Universidade Estadual de Goiás (UEG) e a Fundação de Empreendimentos CientíficoseTecnológicos(FINATEC).

\section{Referências bibliográficas}

1. Baïri, A; Laraqi, N.; Gárcia De María, J. M. Determination of thermal diffusivity of foods using 1D Fourier cylindrical solution. Journal of Food Engineering, v.78, p.669-675, 2007.

2. Baïri, A; Gárcia De María, J. M.; Laraqi, N. Thermal diffusivity measurements of building materials based on 2D models. Computational Materials Science, v.38, p.838-846, 2009.

3. Betta, G.; Rinaldi, M.; Barbanti, D.; Massini, R. A quick method for thermal diffusivity estimation: Application to several foods. Journal of Food Engineering, v.91, p.34-41, 2009. 
4. Boyce, W.E.; Diprima, R.C. Elementary Differential Equations and Boundary Value Problems. New York, John Wiley \& Sons, 2000.

5. Carbonera, L.; Carciofi, B.M.; Huber, E.; Laurindo. Experimental determination of thermal diffusivity in commercial tomato paste. J. B. Braz. J. Food. Technol., v.6, p.285-290, 2003.

6. Carciofi, B.M.; Faistel, J.; Aragão, G.M.F; Laurindo. Determination of thermal diffusivity of mortadella using actual cooking process data. J.B. Journal of Food Engineering, v.55, p.89-94, 2002.

7. Drusas, A.; Saravacos, G.D. Thermal conductivity of tomato paste. J. Food Engineering, v.4, p.157-168, 1985.

8. Erdoğdu, F. Mathematical approaches for use of analytical solutions in experimental determination of heat and mass transfer parameters. Journal of Food Engineering, v.68, p.233-238, 2005.

9. Fourier J. B. J.. Théorie Analytique de la chaleur. Cambridge, 1822.

10. Garrote, R.; Silva, E.; Bertone, R. Effect of thermal treatment on steam peeled potatoes. Journal of Food Engineering, v.45, p.6776, 2000.

11. Huang, L.; Liu, L. Simultaneous determination of thermal conductivity and thermal diffusivity of food and agricultural materials using a transient plane-source method. Journal of Food Engineering, v.95, p.179-185, 2009.

12. Levenberg, K. A method for the solution of certain problems in least squares J. Numer. Anal., v.16, p.588-604, 1944.

13. Marquardt, D. An algorithm for least squares estimation of nonlinear parameters J. Appl. Math., v.11, p.431-441, 1963.
14. Park, K. J.; Murr, F. E. X.; SALVADEGO, M. Medição da condutividade térmica de milho triturado pelo método da sonda. Ciência e tecnologia de alimentos, v.17, n.3, p.242-247, 1997.

15. Silans, A. L.; Da Silva, F. M.; Barbosa, F. A. R. R. Determinação in loco da difusividade térmica num solo da região de caatinga (PB). Bras. Ci. Solo, v.30, p.41-48, 2006.

16. Sousa, T. N. Modelagem matemática para estimativa da difusividade térmica: Experimentação em pasta de pequi e massa de tomate. Anápolis, 2009. Trabalho de conclusão de curso (Graduação em Química Industrial). Universidade Estadual de Goiás (UEG).

\section{Laís S. de Moura', Ana C. Teixeira', Nathália G. Cardoso', Orlene S. Costa', Valter H. C. Silva ${ }^{1,2}$, José S. Sales' \& Heibbe C. B. de Oliveira².}

${ }^{1}$ Unidade Universitária de Ciências Exatas e Tecnológicas, Universidade Estadual de Goiás, P.O. Box 459, 75001-970, Anápolis, GO, Brazil.

${ }^{2}$ Instituto de Química, Universidade de Brasília, 70919-970, Brasília, DF, Brazil.

*e-mail: fatioleg@ueg.br 